Association for Information Systems AIS Electronic Library (AISeL)

Wirtschaftsinformatik Proceedings 2005

Wirtschaftsinformatik

February 2005

\title{
Integration von Business Software - Eine Studie zum aktuellen Stand in Schweizer KMU
}

Uwe Leimstoll

Fachhochschule beider Basel

Petra Schubert

Fachhochschule beider Basel

Follow this and additional works at: http://aisel.aisnet.org/wi2005

\section{Recommended Citation}

Leimstoll, Uwe and Schubert, Petra, "Integration von Business Software - Eine Studie zum aktuellen Stand in Schweizer KMU" (2005). Wirtschaftsinformatik Proceedings 2005. 51.

http://aisel.aisnet.org/wi2005/51

This material is brought to you by the Wirtschaftsinformatik at AIS Electronic Library (AISeL). It has been accepted for inclusion in Wirtschaftsinformatik Proceedings 2005 by an authorized administrator of AIS Electronic Library (AISeL). For more information, please contact elibrary@aisnet.org. 
In: Ferstl, Otto K, u.a. (Hg) 2005. Wirtschaftsinformatik 2005: eEconomy, eGovernment, eSociety; 7. Internationale Tagung Wirtschaftsinformatik 2005. Heidelberg: Physica-Verlag

ISBN: 3-7908-1574-8

(C) Physica-Verlag Heidelberg 2005 


\title{
Integration von Business Software - Eine Studie zum aktuellen Stand in Schweizer KMU
}

\author{
Uwe Leimstoll, Petra Schubert \\ Fachhochschule beider Basel
}

Zusammenfassung: Die Integration von Informationssystemen ist ein Thema, das in der Literatur schon lange diskutiert wird. Aufgrund der technologischen Entwicklung haben sich die Voraussetzungen für Integration in den letzten Jahren erheblich verbessert. Der vorliegende Beitrag konzentriert sich auf die Integration von Business Software in KMU und zeigt anhand empirischer Primärdaten den aktuellen Stand in der Schweiz auf. Dabei wird unter anderem deutlich, welche Erfahrungen in bisherigen Integrationsprojekten gemacht wurden und welche Anforderungen an zukünftige Business-Software-Lösungen gestellt werden. Es zeigt sich, dass bei einigen Software-Modulen die Intensität der Nutzung und der Grad der Integration von der Unternehmensgrösse abhängig ist.

Schlüsselworte: Business Software, E-Business, Integration, KMU

\section{Einführung}

Der folgende Beitrag beschäftigt sich mit dem State-of-the-Art der Integration von Business Software und E-Business-Applikationen in der unternehmerischen Praxis. Um ein Bild vom aktuellen Stand des Einsatzes in Schweizer KMU zu erhalten, wurde im Herbst 2003 eine repräsentative Studie durchgeführt [Det ${ }^{+}$04]. Darin wurde bestätigt, dass die Unterstützung von Geschäftsprozessen mit Business Software mittlerweile auch in kleinen und mittleren Unternehmen weit verbreitet ist. Die am Markt verfügbaren Lösungen werden zunehmend in modularer Form angeboten und erfüllen sowohl leistungs- als auch kostenseitig immer mehr die besonderen Anforderungen kleinerer Unternehmen. Es zeigt sich eine starke Konkurrenz unter sehr vielen alternativen Anbietern, was zu einer starken Ausrichtung auf individuelle Kundenbedürfnisse führt. Diese Situation hat in der Schweiz eine heterogene Angebotsvielfalt entstehen lassen, die neben Standardsoftwarepaketen auch Nischen für spezialisierte Lösungen zulässt.

Ein zentrales Thema, das im Zusammenhang mit der informationstechnischen Unterstützung von Geschäftsprozessen schon seit längerer Zeit in der Literatur disku- 
tiert wird, ist das Thema der Integration. Die Ende der 80er und Anfang der 90er Jahre beschriebenen CIM-, CIB- und CIE-Konzepte ${ }^{1}$ können als gescheitert bezeichnet werden. Zumindest für KMUs waren diese Konzepte nicht geeignet. Seinerzeit fehlten zum einen geeignete technische Systeme, zum anderen aber auch eine ausreichende Ausrichtung der organisatorischen und systemtechnischen Integration am Bedarf der Unternehmen [Sche96, S. 255; Dürs98, S. 97].

Integrierte Lösungen blieben damit lange Zeit zunächst grösseren Unternehmen vorbehalten. Mit der zunehmenden technischen Entwicklung und dem Trend zu einer mehr prozessorientierten Organisationsgestaltung ebnete sich ab Mitte der 90er Jahre der Weg zu mehr Vernetzung und Integration. Diese Entwicklung betraf zunächst überwiegend unternehmensinterne Anwendungen (z. B. EnterpriseResource-Planning(ERP)-Systeme) und dehnte sich später auf unternehmensübergreifende Applikationen (z. B. E-Business-Systeme) aus.

Bis heute haben sich die technischen und organisatorischen Bedingungen für eine Integration von Informationssystemen deutlich verbessert. Zum einen nahm die für die Übertragung der Daten nötige Bandbreite erheblich zu, womit sich die Übertragungsrate bei gleichzeitig sinkenden Kosten erheblich erhöhte. Mit der Verbreitung der Internetprotokolle und weiterer Standards zum Austausch von Daten (XML, SOAP, etc.) entstand eine wichtige Basis für unternehmensübergreifende Anwendungen. Heute existieren im Bereich der Business Software viele Anwendungen mit integrierten E-Business-Modulen. Weitere Schritte zur direkten B2B-Integration heterogener ERP-Systeme sind im Gange.

Die Entscheidung, bis zu welchem Grad Business Software integriert werden soll, ist für kleine und mittlere Unternehmen auch heute noch schwierig. Den vermeintlichen ökonomischen Vorteilen, wie die Vermeidung von Medienbrüchen und eine höhere Kundenbindung, stehen gewichtige Nachteile gegenüber. Letztere entstehen in erster Linie aufgrund der Komplexität integrierter Systeme. Die Probleme der Systemkomplexität kommen in KMU besonders zum Tragen: häufig fehlen das nötige Know-how oder die finanziellen Ressourcen, um Integrationsprojekte zu realisieren. Auch der Return on Investment fällt in kleineren Unternehmen aufgrund der niedrigeren Transaktionshäufigkeit tendenziell niedriger aus als in grösseren Unternehmen [Leim01, S. 233 f.].

Vor diesem Hintergrund soll der vorliegende Beitrag aufzeigen, welche Erfahrungen Schweizer KMU in Integrationsprojekten gemacht haben und welche Erwartungen sie mit zukünftigen Anwendungen verknüpfen. Im Zentrum der Diskussion stehen die Ergebnisse einer empirischen Studie [Det ${ }^{+}$04]. Auf der Grundlage theoretischer Überlegungen werden darüber hinaus Hypothesen formuliert und getestet, die Aussagen zu Abhängigkeiten zwischen Unternehmensgrösse, Umsatzentwicklung, Integrationsgrad und Unternehmenserfolg machen.

$\mathrm{CIM}=$ Computer Integrated Manufacturing; $\mathrm{CIB}=$ Computer Integrated Business; $\mathrm{CIE}=$ Computer Integrated Enterprise [Sche91] 
Der vorliegende Beitrag gibt zunächst einen kurzen Überblick über Begriffe und theoretische Grundlagen zum Thema Integration von Business Software und EBusiness-Applikationen. Danach werden die Untersuchungsziele näher spezifiziert und Hypothesen formuliert. Kapitel 4 beschreibt das Design der empirischen Studie. Im Anschluss daran werden ausgewählte Ergebnisse einer deskriptiven Analyse vorgestellt, bevor die Hypothesen getestet werden. Eine Zusammenfassung schliesst den Beitrag ab.

\section{Integration und Business Software}

Die Frage nach dem optimalen Grad an E-Business-Integration ist ein fundamentales Thema der Wirtschaftsinformatik. Wie Davydov in seiner folgenden Aussage deutlich macht, ist E-Business auf das Herstellen von Verbindungen sowohl zwischen Unternehmensbereichen als auch zwischen Geschäftspartnern ausgerichtet:

Fundamentally, e-business is, first and foremost, about breaking all kinds of „walls“ - internal corporate „walls“ that exist between functional departments, but more importantly, external "walls" that limit companies' willingness and actual abilities to engage in new business relationships and accept new ideas. [Davy01, S. 17]

Im Rahmen des vorliegenden Beitrags wird in Erweiterung dieses Zitats die folgende Definition des Begriffs „E-Business-Integration“ zugrunde gelegt:

E-Business-Integration ist die Verbindung von Geschäftsprozessen und Informationssystemen mit dem Ziel, in einer verteilten Wertschöpfungskette eine zusammenhängende Leistung (für den Kunden) zu erzeugen.

Die Integration von Informationssystemen kann auf verschiedenen Ebenen vollzogen werden: auf der Präsentationsebene, der Applikationsebene und der Datenebene. Dabei wird zwischen interner und externer Integration unterschieden. Die Applikationssicht der internen Integration wird in der Literatur unter dem Stichwort „Enterprise Application Integration (EAI)“ behandelt und betrachtet die Integration von Informationssystemen innerhalb eines Unternehmens [Davy01; $\mathrm{Buh}^{+}$01; Dett02; ScWi02; Kell02; Kaib02; VoZe02; Holt03]. Das entsprechende Pendant dazu, die Applikationssicht der externen Integration, wird unter dem Begriff „B2B Application Integration (BBAI)“ diskutiert und ist in der Praxis noch wenig verbreitet [Lint01; ScDo02]. Ihr Ziel ist es, durch den Einsatz von definierten Formaten und Protokollen Unternehmensgrenzen zu überbrücken. Beispiele hierfür sind die Integration eines E-Shops in die E-Procurement-Lösung des Kunden oder die automatische Übertragung von Auftrags- und Konfigurationsdaten in die Auftragsbearbeitung und Produktionsplanung des Lieferanten.

Abbildung 1 zeigt einen systematischen Überblick über Managementkonzepte, Applikationen und involvierte Parteien. Dabei steht die Betrachtung eines konkreten Unternehmens im Zentrum (skizziert durch die gestrichelte Linie). Das Unter- 
nehmen verfügt über ein ERP-System, mit dessen Hilfe verschiedene Aufgaben der Unternehmensführung wie Einkauf, Verkauf und Rechnungswesen unterstützt werden. Eine detaillierte Beschreibung der aufgeführten Begriffe findet sich in [Det 04$]$.

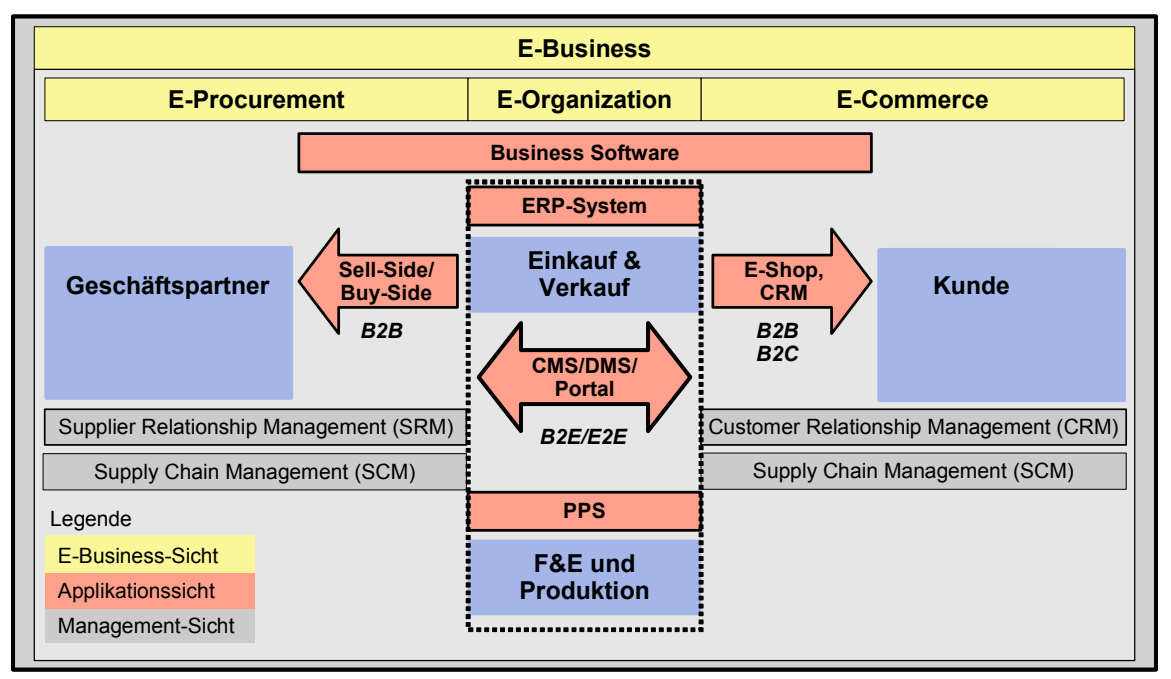

Abbildung 1: Begriffssystematik: ERP und E-Business; Quelle: [Schu04, S. 2]

Die folgenden Ausführungen befassen sich schwerpunktmässig mit ERPSystemen und damit verbundenen E-Business-Applikationen. In diesem Kontext bezeichnen wir die Kopplung von Informationssystemen als Integration, also z. B. eine Verbindung verschiedener ERP-Module wie Buchhaltung und Bestellwesen oder die Kopplung der ERP-Auftragsverwaltung mit einem E-Business-Modul wie etwa einem E-Shop [Sch ${ }^{+}$03].

Der Begriff Business Software dient als Überbegriff für betriebswirtschaftliche Software. Sie schliesst damit sowohl ERP-Software als auch E-Business-Software ein. ERP-Software-Basismodule unterstützen in erster Linie die unternehmensinternen Funktionsbereiche und Geschäftsprozesse. Finanzbuchhaltung, Warenwirtschaft, Einkauf und Vertrieb bilden meist die Kernbereiche für den Einsatz von ERP-Systemen. E-Business-Software-Module unterstützen schwerpunktmässig die unternehmensübergreifenden Geschäftsprozesse. Tabelle 1 gibt einen Überblick über die untersuchten Software-Module.

\begin{tabular}{ll}
\hline $\begin{array}{l}\text { ERP-Module } \\
\text { (unternehmensintern) }\end{array}$ & $\begin{array}{l}\text { E-Business-Module } \\
\text { (unternehmensübergreifend) }\end{array}$ \\
\hline $\begin{array}{l}\text { Finanzwirtschaft (Debitoren, Kredito- } \\
\text { ren, FiBu, KoRe, AnBu) }\end{array}$ & E-Procurement (Beschaffung) \\
\hline Warenwirtschaft (Logistik und Lager- & E-Organization (Collaboration, Projektmana- \\
\hline
\end{tabular}




\begin{tabular}{ll}
\hline haltung) & gement, Leistungserfassung und -verrechnung) \\
\hline Einkauf & E-Commerce (E-Shop) \\
\hline Vertrieb und Auftragsabwicklung & $\begin{array}{l}\text { Marketing und Customer Relationship Mana- } \\
\text { gement (CRM) }\end{array}$ \\
\hline $\begin{array}{l}\text { Service und Dienstleistungen } \\
\text { (Leistungs- und Projektabrechnung) }\end{array}$ & Supply Chain Management (SCM) \\
\hline Produktionsplanung und -steuerung & $\begin{array}{l}\text { Mobile Applikationen (z. B. mobiler Zugriff } \\
\text { auf Produktkataloge, Kundendaten; mobile }\end{array}$ \\
\hline Buftragserfassung)
\end{tabular}

Tabelle 1: Untersuchte Software-Module

\section{Gegenstand der Untersuchung und Hypothesenbildung}

Um den aktuellen Stellenwert der Business-Software-Integration in Schweizer KMU abbilden zu können, gilt es zunächst zu erfassen, welche Verbreitung ERPund E-Business-Software in KMU gefunden hat und welche Bedeutung der internen und externen Integration heute zukommt. Die Erfahrungen aus bisherigen Projekten können aufzeigen, welche Schwierigkeiten bisher beobachtet worden sind.

Um Aussagen über die zukünftige Entwicklung machen zu können, sollen die Ziele erfasst werden, die Schweizer KMU anstreben, wenn sie künftig Integrationsprojekte in Angriff nehmen. Für die Software-Branche dürfte vor allem interessant sein, welche Anforderungen in diesem Zusammenhang an künftige Systeme gestellt werden.

Neben der beschreibenden Analyse sollen im Folgenden Faktoren ermittelt werden, die den Einsatz und die Integration von Business Software in KMU beeinflussen. Zudem sollen Aussagen darüber gemacht werden, ob Einsatz und Integration von Business Software positive Auswirkungen auf den Unternehmenserfolg haben.

In einem ersten Schritt bilden die Unternehmensgrösse und die Umsatzentwicklung als Beispiele für Charakteristika von Anwenderunternehmen exogene Fakto- 
ren. Damit wird ergründet, ob organisationale Eigenschaften einen Einfluss auf die Nutzung von Business Software und den Grad der Integration haben. Die Unternehmensgrösse (Anzahl der Mitarbeitenden) erscheint als Einflussfaktor sehr plausibel. Mit steigender Mitarbeiterzahl nehmen die betrieblichen Aufgaben zu, was einen höheren Abwicklungs- und Steuerungsaufwand verursacht [Leim01, S. 405 f.]. Damit wächst der Bedarf an unterstützenden Informationssystemen.

Als weiterer exogener Faktor wird die Umsatzentwicklung herangezogen. Ein steigender Umsatz erhöht in der Regel die Transaktionshäufigkeit und begünstigt meist den Cashflow. Diese Effekte könnten sich positiv auf den Nutzen integrierter Business Software auswirken.

In einem zweiten Schritt bilden der Einsatz und die Integration von Business Software die exogenen Faktoren, um ihren Einfluss auf den Unternehmenserfolg zu prüfen. Der Einsatz von Informationssystemen als erfolgswirksamer Faktor in KMU wird bisher durch mehrere Studien belegt [Ray ${ }^{+}$95, S. 10 f.; Lyba96, S. 52; $\mathrm{Sch}^{+}$93; LePo98, S. 193; Leim01, S. 445 ff.]

Abbildung 2 zeigt die beschriebenen Zusammenhänge grafisch auf. Sechs Hypothesen lassen sich auf dieser Basis formulieren. Die Nummern in der Abbildung beziehen sich auf die Nummerierung der folgenden Hypothesen. Alle unterstellten Zusammenhänge sind positiv.

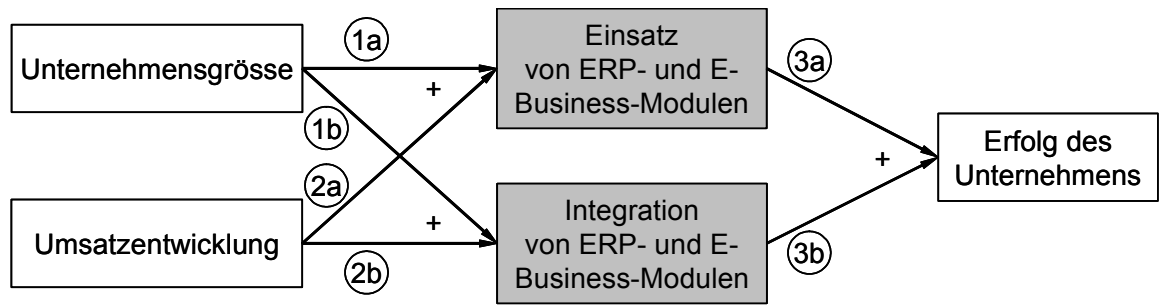

Abbildung 2: Wirkungsrichtung der unterstellten Zusammenhänge

Hypothese 1a: Grössere Unternehmen setzen häufiger ERP- und E-BusinessModule ein als kleinere Unternehmen.

Hypothese 1b: In grösseren Unternehmen werden diese Systeme häufiger integriert als in kleineren Unternehmen.

Hypothese 2a: Je mehr im Unternehmen der Eindruck entsteht, die Umsatzentwicklung verlaufe positiv, desto mehr sind die Unternehmen bereit, ERP- und EBusiness-Systeme einzusetzen.

Hypothese 2b: Je mehr im Unternehmen der Eindruck entsteht, die Umsatzentwicklung verlaufe positiv, desto mehr sind die Unternehmen bereit, ERP- und EBusiness-Systeme zu integrieren. 
Hypothese 3a: Unternehmen, die in grösserem Umfang ERP- und E-BusinessSysteme einsetzen, verzeichnen einen grösseren Unternehmenserfolg als andere Unternehmen.

Hypothese 3b: Unternehmen, die in grösserem Umfang ERP- und E-BusinessSysteme zu einem Gesamtsystem integrieren, verzeichnen einen grösseren Unternehmenserfolg als andere Unternehmen.

\section{Forschungsdesign}

\subsection{Untersuchungsmethodik}

Zur Erhebung der Primärdaten wurde im Herbst 2003 eine schriftliche Umfrage durchgeführt. Dazu wurden 5'032 Schweizer Unternehmen und andere Organisationen mit 10 bis 250 Beschäftigten in den Wirtschaftssektoren zwei (Industrie) und drei (Dienstleistungen) befragt. Das Schweizer Bundesamt für Statistik zog eine zufällige, nach Branche und Unternehmensgrösse geschichtete Stichprobe aus der Grundgesamtheit der über 33'500 KMU (Abbildung 3).

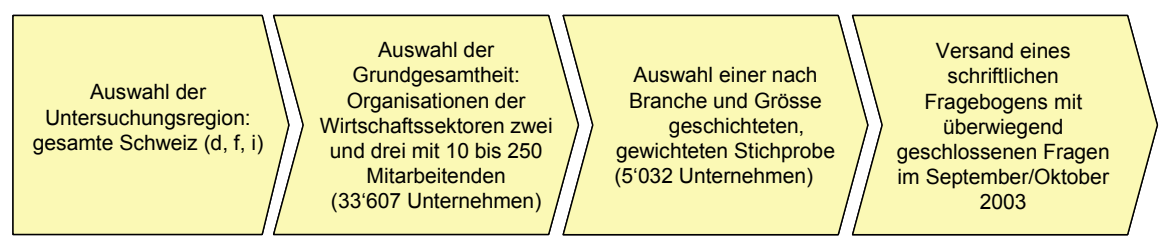

Abbildung 3: Vorgehen bei der Datenerhebung

In Zusammenarbeit mit industriellen Forschungspartnern wurde ein standardisierter Fragebogen entwickelt und in Pretest-Interviews mehrfach geprüft. Er richtete sich an Mitglieder der Geschäftsleitung. Wegen der weiten Verbreitung des Internets in der Schweiz wurde zusätzlich zum gedruckten Fragebogen eine OnlineVersion des Fragebogens zur Verfügung gestellt.

Insgesamt antworteten 819 Unternehmen (16.3\%) auf den erhaltenen Fragebogen. Darunter waren viele Absagen, die häufig auch begründet wurden. $15.5 \%$ der absagenden Unternehmen haben z. B. keine Business Software im Einsatz.

494 Unternehmen sandten einen ausgefüllten Fragebogen ein, was einer Rücklaufquote von $9.8 \%$ entspricht. 463 dieser Fragebogen konnten letztlich für die Auswertung verwendet werden, was einer Netto-Rücklaufquote von $9.2 \%$ entspricht. Tabelle 2 fasst die Eckdaten der Datenerhebung zusammen. 


\begin{tabular}{ll}
\hline Erhebungszeitraum & September/Oktober 2003 \\
\hline Erhebungsregion & gesamte Schweiz (Sprachregionen D, F, I) \\
\hline Erhebungsmethode & $\begin{array}{l}\text { schriftliche Umfrage mit standardisiertem Fragebogen (ein Print- } \\
\text { Fragebogen wurde verschickt, zusätzlich stand ein Online- } \\
\text { Fragebogen zur Verfügung) }\end{array}$ \\
\hline Unternehmensgrösse & KMU mit 10 bis 250 Beschäftigten (Vollzeitäquivalent) \\
\hline Branche & Wirtschaftssektoren zwei (Industrie) und drei (Dienstleistungen) \\
\hline Zielgruppe & Geschäftsführer und Informatik-Verantwortliche in KMU \\
\hline Stichprobe & 5'032 Unternehmen, geschichtet nach Unternehmensgrösse und \\
\hline Rücklauf & Branche \\
\hline
\end{tabular}

Tabelle 2: Eckdaten der empirischen Erhebung im Überblick

Der Anteil kleiner Unternehmen ist in der Grundgesamtheit deutlich höher als in der Stichprobe. Die Daten für die deskriptive Auswertung werden deshalb nach der Unternehmensgrösse gewichtet und dürfen für Schweizer KMU, die ERPSysteme einsetzen, als insgesamt repräsentativ betrachtet werden.

\subsection{Charakterisierung der Stichprobe}

Aufgrund der inhaltlich anspruchsvollen Fragestellung sollte davon ausgegangen werden, dass sich verstärkt solche Unternehmen an der Umfrage beteiligten, die sich bereits intensiv mit den Themen „ERP-Systeme“ und „E-Business-Integration" auseinander gesetzt hatten. Um einen Eindruck von der Zusammensetzung der vorliegenden Stichprobe zu vermitteln, beschreiben die nächsten Abschnitte einige wesentliche Eigenschaften der Unternehmen.

\section{Unternehmen nach Unternehmensgrösse}

Die Verteilung der Unternehmen nach Unternehmensgrössenklassen zeigt ein recht gleichmässiges Bild. Die Unternehmensgrösse wurde an der Anzahl der Beschäftigten gemessen, wobei das Vollzeitäquivalent erfasst wurde. Der Hauptanteil der Unternehmen in der Stichprobe (39\%) beschäftigt zwischen 10 und 49 Mitarbeitende (MA). $28 \%$ der Unternehmen beschäftigen zwischen 50 und 99 Mitarbeitende, $33 \%$ zwischen 100 und 250 Mitarbeitende (Abbildung 4).

Die Grössenverteilung in der Stichprobe entspricht damit nicht der Verteilung in der Grundgesamtheit, in der die kleineren Unternehmen einen wesentlich grösseren Anteil ausmachen. Die Ergebnisse der deskriptiven Analyse basieren deshalb auf Daten, die nach der Unternehmensgrösse gewichtet wurden. So lassen sich repräsentative Aussagen über die KMU in der Schweiz machen. 


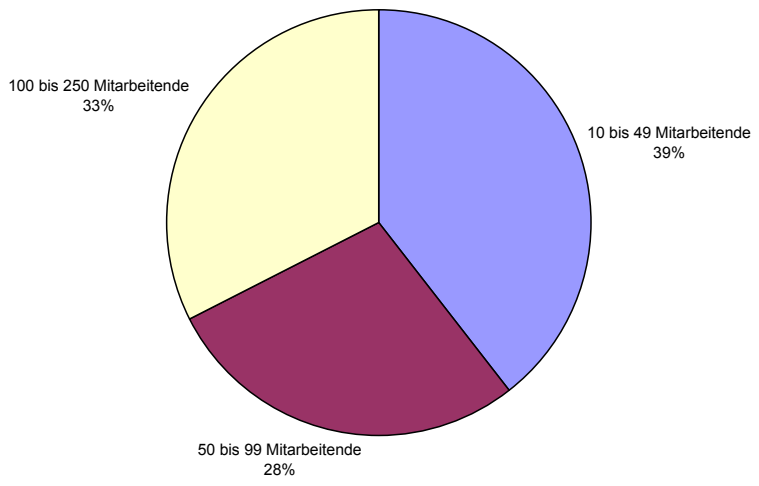

Abbildung 4: Unternehmen in der Stichprobe nach Anzahl der Beschäftigten, N=424

\section{Unternehmen nach Branche}

In der Stichprobe sind Unternehmen der Wirtschaftssektoren zwei (Industrie) und drei (Dienstleistungen), also fast aller Branchen vertreten. Den grössten Anteil macht das Gesundheits- und Sozialwesen aus (17\%), dicht gefolgt vom Verarbeitenden Gewerbe und der Industrie (16\%) sowie den Sonstigen Dienstleistungen (16\%). Auch das Baugewerbe (15\%) und die Öffentliche Verwaltung einschliesslich des Unterrichtswesens (13\%) sind recht stark vertreten (Abbildung 5). In den betrachteten drei Unternehmensgrössenklassen zeigt die Branchenverteilung ein recht einheitliches Bild.

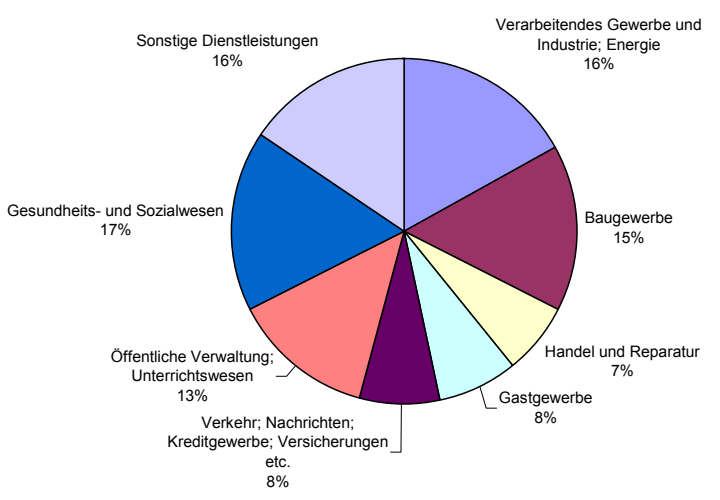

Abbildung 5: Unternehmen in der Stichprobe nach Branche, $\mathrm{N}=442$ 


\section{Deskriptive Auswertung}

$60 \%$ der Schweizer KMU äussern, dass die Kosten von Integrationsprojekten für sie zu hoch seien. Diese Aussage resultiert aus den Erfahrungen mit bisherigen Projekten zur Integration von Informationssystemen. Beobachtet wurde ausserdem, dass durch Integration zu viele Schnittstellen entstehen (48 \%). Ein weiterer kritischer Punkt bildet die Nutzenproblematik, wobei hier zwischen der Höhe und der Bewertung des Nutzens unterschieden wird. Für fast die Hälfte der KMU lässt sich zum einen der Nutzen der Integration im Voraus nicht eindeutig bewerten und zum anderen erscheint der Nutzen von Integrationsprojekten als zu gering (Abbildung 6).

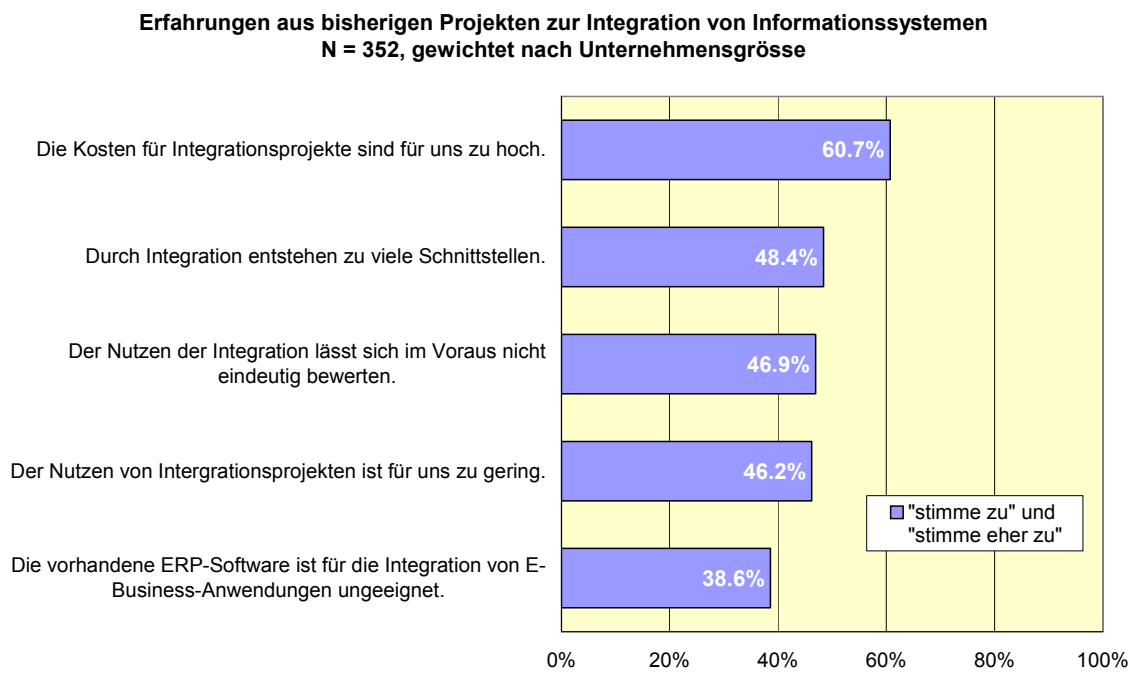

Abbildung 6: Erfahrungen aus bisherigen Projekten zur Integration von Informationssystemen

Welchen Nutzen kann die Integration von Business Software nun konkret generieren? Auf der Datenebene stehen der Zugriff auf eine einheitliche Datenbasis und die medienbruchfreie Übertragung der Daten zwischen verschiedenen Applikationen oder Systemmodulen im Vordergrund. Auf der organisatorischen Ebene werden die Beschleunigung und die qualitative Verbesserung funktionsübergreifender Prozesse hervorgehoben [Dürs98]. Bei der unternehmensübergreifenden Integration kommen ausserdem strategische Vorteile, wie etwa eine höhere Kundenbindung, hinzu.

Die Schweizer KMU bringen zunächst zum Ausdruck, dass sie die Bedeutung von integrierten Systemen in stärkerem Masse in der Unterstützung unternehmensinterner Prozesse sehen. Rund 80 \% der KMU sagen aus, dass integrierte Systeme 
zur Abwicklung von Transaktionen innerhalb des Unternehmens wichtig seien und künftig die interne Zusammenarbeit wesentlich erleichterten. Demgegenüber sagen gut $60 \%$ der KMU aus, dass integrierte Systeme zur Abwicklung von Transaktionen über die Unternehmensgrenzen hinweg wichtig seien und künftig die Zusammenarbeit mit Kunden und Lieferanten wesentlich erleichterten (Abbildung 7).

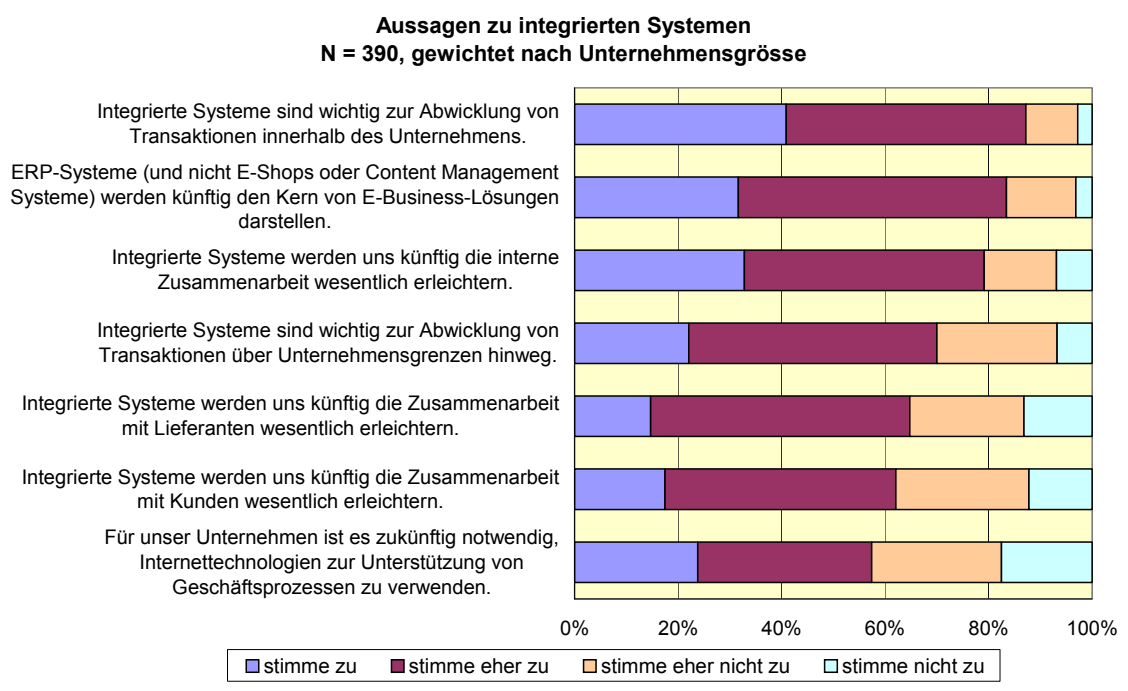

Abbildung 7: Aussagen zu integrierten Systemen

Der Grund, dass die Integration von Business Software mehr auf die unternehmensinternen Prozesse abzielen wird, mag darauf zurückzuführen sein, dass KMU den unternehmensinternen Bereich in stärkerem Masse mit Business Software unterstützen. ERP-Software-Basismodule werden heute im Durchschnitt jeweils von etwa der Hälfte der KMU eingesetzt. E-Business-Software-Module kommen demgegenüber im Durchschnitt lediglich jeweils auf knapp $10 \%$. Letztere werden künftig allerdings eine deutliche Zunahme verzeichnen: In den kommenden zwei Jahren wird sich der Einsatz von E-Business-Software-Modulen in Schweizer KMU durchschnittlich verdoppeln [Det $\left.{ }^{+} 04\right]$.

Worin liegt nun im Einzelnen der Nutzen, den Schweizer KMU zukünftig mit Integrationsprojekten erzielen möchten? Über die nächsten zwei Jahre betrachtet wird hier die Erhöhung der Datenqualität genannt, fast gleichauf mit technischen Aspekten (Reduktion der Schnittstellen, Reduktion des Wartungsaufwandes, Reduktion von Datenredundanzen) und der Vereinheitlichung der Informationssysteme. Diese drei informatikorientierten Aspekte stehen mit jeweils rund $80 \% \mathrm{Zu}-$ stimmung im Vordergrund. Sie werden gefolgt von drei mehr betriebswirtschaftlich ausgerichteten Nutzenaspekten, die jeweils rund 70 \% Zustimmung erhalten: 
die Beschleunigung der Auftragsabwicklung, die Reduktion der Auftragsabwicklungskosten und die Steigerung der Produktivität (Abbildung 8).

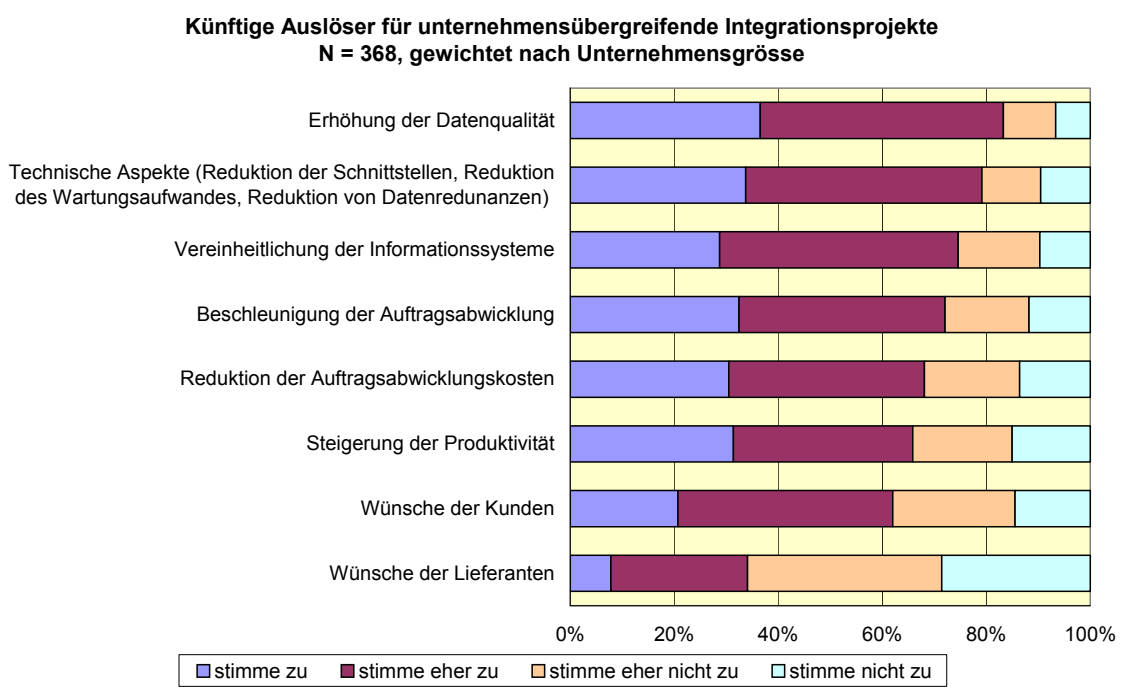

Abbildung 8: Künftige Auslöser für die Durchführung von Integrationsprojekten

Etwas überraschend fällt demgegenüber die Erfüllung von Kundenwünschen ab. $60 \%$ der KMU geben hier ihre Zustimmung, dass sie Integrationsprojekte künftig in Angriff nehmen, um die Wünsche der Kunden zu erfüllen. Diese Einschätzung passt wiederum zum bereits erwähnten stärker unternehmensintern orientierten Fokus der Integration. Ebenfalls passt dazu die Beobachtung, dass die Wünsche der Lieferanten eine eher untergeordnete Rolle spielen (Abbildung 8).

Damit die Integration von Informationssystemen den gewünschten Nutzen erzielt und nicht gleichzeitig durch eine höhere Komplexität der Systeme für KMU zu unhandlich oder zu teuer wird, sind geeignete Systeme erforderlich. Genau genommen handelt es sich um ERP-Systeme, die Erweiterungen für den E-BusinessBereich optional bereithalten. $84 \%$ der KMU, die dazu eine Aussage machen können, tendieren zu der Meinung, dass ERP-Systeme künftig den Kern von EBusiness-Lösungen bilden werden (Abbildung 7).

Die Anforderungen an künftige ERP-Systeme sind auf Seiten der KMU sehr hoch. Unter den kaufentscheidenden Kriterien steht die Abdeckung von Schweizer Bedürfnissen (Vorschriften für Rechnungslegung, Lohnabrechnung, Buchhaltung, etc.) an erster Stelle. Mehr als die Hälfte der Schweizer KMU bezeichnet dieses Kriterium als „kaufentscheidend“. An zweiter Stelle folgen Flexibilität und Releasefähigkeit der Systeme, gefolgt von der kurzen Reaktionszeit des Anbieters oder seines Support-Partners im Support-Fall und der Erweiterbarkeit der Systeme. Berücksichtigt man zur Einstufung „kaufentscheidend“ noch die Bewertung 
„wichtig“, dann erreicht jede dieser vier Anforderungen über $90 \%$ der Nennungen (Abbildung 9). Demzufolge spielen die Aspekte der Weiterentwicklung und der Wartung der Systeme neben der Abdeckung der Schweizer Bedürfnisse eine herausragende Rolle.

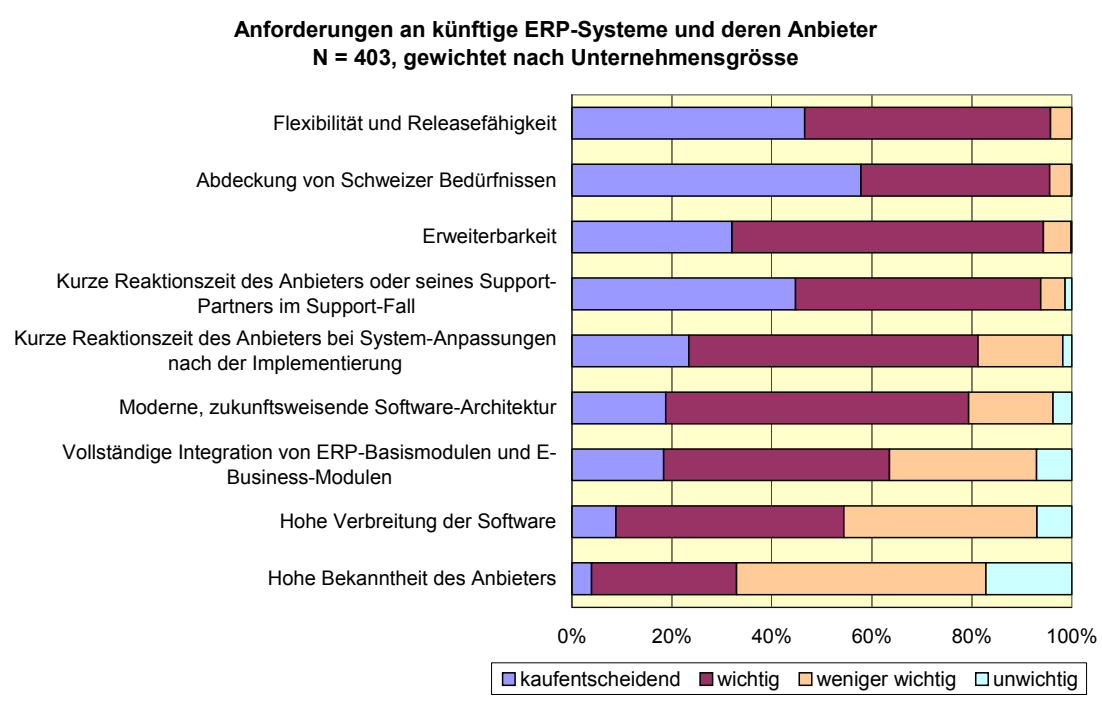

Abbildung 9: Anforderungen an künftige ERP-Systeme und deren Anbieter

Abbildung 9 zeigt einige weitere Anforderungen an ERP-Systeme, die zum Teil auch recht häufig als ,wichtig“ eingestuft werden. Dies trifft zum Beispiel auf die vollständige Integration von ERP-Basismodulen und E-Business-Modulen zu. Die Bewertungen „kaufentscheidend“ und „wichtig“ zusammengenommen, kommt dieses Kriterium auf $60 \%$ der Nennungen. Dies bestätigt wiederum die These, dass ERP-Systeme eine zentrale Grundlage von E-Business-Lösungen bilden und Einführung und Integration von E-Business-Lösungen künftig zunehmen werden.

Wie eingangs schon erwähnt, zeichnet sich der Schweizer Markt für Business Software durch eine grosse Heterogenität aus. Selbst der Marktführer Abacus kommt nur auf einen Marktanteil in Höhe von etwa $16 \%$. Den Rest teilen sich mehr als 200 verschiedene Hersteller und Händler. ${ }^{2}$ Neben den eher kleinen, aber sehr wettbewerbsfähigen Schweizer Anbietern tummeln sich die Tochterfirmen der international bekannten Anbieter auf diesem Markt. Einige Anbieter haben sich auf die Anforderungen einzelner Branchen spezialisiert und innerhalb dieser Nischen etabliert. Für den Anwender stellt sich ob dieser Vielfalt die Frage, welcher Anbieter nun der Richtige für ihn wäre.

2 Genaue Zahlen und die Namen der führenden Anbieter finden sich in [Det ${ }^{+} 04, \mathrm{~S}$. $15 \mathrm{ff}$.$] .$ 
Besonderen Wert legen die Schweizer KMU auf die Offenheit der SoftwareModule, damit die Module beliebiger Hersteller miteinander kombiniert werden können. Auch darin steckt wiederum ein Hinweis auf die wachsende Bedeutung der Integration und auf den Wunsch nach Flexibilität. Neben der Offenheit der Systeme wird gleichzeitig gewünscht, alle Software-Module eines ERP-Systems von demselben Anbieter zu beziehen. Dies wiederum unterstreicht das Bedürfnis der KMU nach einer einfachen und direkten Koordination mit dem Anbieter, derso die überwiegende Meinung - möglichst in der Nähe des Anwenders niedergelassen sein sollte. Die besondere Bedeutung regionaler Händler und Integratoren wird darin wieder sichtbar (Abbildung 10).

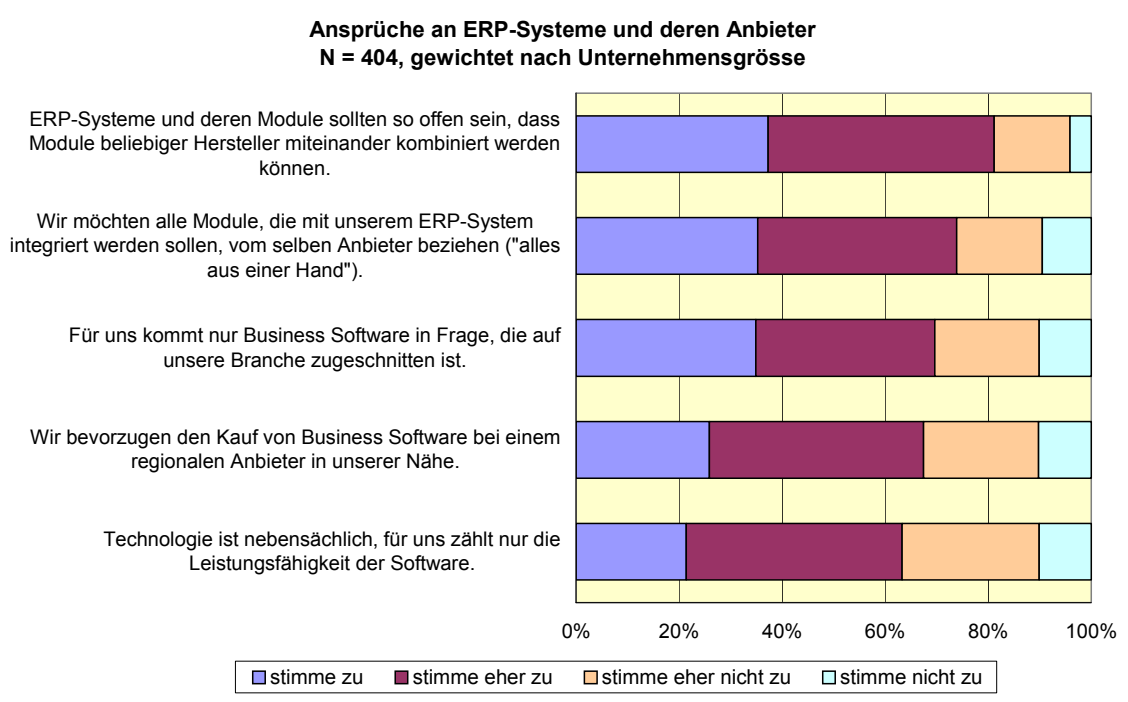

Abbildung 10: Aussagen zu ERP-Systemen und deren Anbieter

\section{Analytische Auswertung}

Für den Test der sechs formulierten Hypothesen liegen kategoriale Daten vor, die sich in Kontingenztafeln übersichtlich darstellen lassen [Stei93, S. 11 ff.]. Zur statistischen Analyse der Kontingenztafeln wird der Chi-Quadrat-Test nach Pearson angewendet [z. B. Bac ${ }^{+}$96; Sach92]. Er erlaubt qualitative Aussagen über die Ab- 
hängigkeit zwischen zwei Merkmalen (Unabhängigkeitstest) sowie die Ermittlung des zugehörigen Signifikanzniveaus. ${ }^{3}$

\section{Hypothese 1}

Hypothese 1 untersucht den Zusammenhang zwischen der Unternehmensgrösse und dem Einsatz und der Integration von ERP- und E-Business-Modulen. Die Unternehmensgrösse wird an der Anzahl der Mitarbeitenden gemessen. Die Antworten werden in die drei Klassen (1) 10 - 49, (2) 50 - 99 und (3) 100 - 250 Mitarbeitende zusammengefasst. Einsatz und Integration der ERP- und E-BusinessModule werden daran gemessen, ob das betreffende Modul vorhanden/integriert ist oder nicht. Dabei werden die Module untersucht, die in Tabelle 1 aufgeführt sind.

Im Falle der Unternehmensgrösse als Determinante des Einsatzes (Hypothese 1a) von ERP-Modulen kann der Chi-Quadrat-Test auf alle Module angewendet werden. Tabelle 3 zeigt, dass ein signifikanter Zusammenhang mit der Unternehmensgrösse für den Einsatz der ERP-Module Vertrieb und Auftragsabwicklung, Warenwirtschaft und Einkauf beobachtet werden kann. Diese Module werden in grösseren Unternehmen häufiger eingesetzt als in kleineren Unternehmen.

Für den Einsatz von E-Business-Modulen können keine signifikanten Zusammenhänge nachgewiesen werden. Die Nullhypothese der Unabhängigkeit ist für diese Module aufrechtzuerhalten. In den meisten Fällen - dies gilt auch für die weiteren Hypothesen - kann der Test wegen zu geringer Erwartungshäufigkeiten nicht angewendet werden. Die geringen Erwartungshäufigkeiten resultieren aus der Tatsache, dass E-Business-Module in den untersuchten KMU (noch) nicht weit verbreitet sind und vergleichsweise wenige Unternehmen Aussagen zu der zugrunde liegenden Frage nach dem Einsatz und der Integration der E-Business-Module machen.

Der Chi-Quadrat-Test ist nur anwendbar auf den Zusammenhang zwischen der Unternehmensgrösse und dem Einsatz der E-Business-Module E-Commerce, Marketing und CRM, Mobile Applikationen, Content Management und Branchensoftware. Ein statistischer Zusammenhang liegt nicht vor, der Einsatz dieser Module ist von der Unternehmensgrösse unabhängig.

Die Unternehmensgrösse bestimmt zum Teil auch den Grad der Integration (Hypothese 1b) der ERP-Module. Dies ist der Fall bei den Modulen Finanzwirtschaft, Warenwirtschaft, Einkauf sowie Controlling und FIS. Diese Module sind

3 Von einem signifikanten Zusammenhang wird dann ausgegangen, wenn die Irrtumswahrscheinlichkeit $\alpha=0.05$ oder weniger beträgt. Dies entspricht einem geforderten Signifikanzniveau von $5 \%$. 
in grösseren Unternehmen häufiger integriert anzutreffen als in kleineren Unternehmen.

Der Einfluss der Unternehmensgrösse auf die Integration der E-Business-Module lässt sich wegen zu geringer Erwartungshäufigkeiten nur für die Module $E$ Commerce, Marketing und CRM und Branchensoftware untersuchen. Diese Zusammenhänge sind nicht signifikant. Die Unternehmensgrösse hat folglich keinen Einfluss darauf, ob E-Commerce-, Marketing- und CRM- und BranchensoftwareModule in ein Gesamtsystem integriert werden oder nicht.

\begin{tabular}{|c|c|c|c|c|c|}
\hline $\begin{array}{l}\text { Hypo- } \\
\text { these }\end{array}$ & Merkmal 1 & Merkmal 2 & Modul & $\begin{array}{l}\text { Signifi- } \\
\operatorname{kanz} * \text { ) }\end{array}$ & $\begin{array}{l}\text { Signifi- } \\
\text { kanz- } \\
\text { niveau } \\
* *)\end{array}$ \\
\hline 1a & $\begin{array}{l}\text { Unterneh- } \\
\text { mensgrösse }\end{array}$ & $\begin{array}{l}\text { Einsatz von } \\
\text { ERP-Modulen }\end{array}$ & $\begin{array}{l}\text { Vertrieb und } \\
\text { Auftragsabwicklung }\end{array}$ & 0.008 & $1.0 \%$ \\
\hline $1 \mathrm{a}$ & & & Warenwirtschaft & 0.010 & $1.0 \%$ \\
\hline $1 \mathrm{a}$ & & & Einkauf & 0.043 & $5.0 \%$ \\
\hline $1 b$ & $\begin{array}{l}\text { Unterneh- } \\
\text { mensgrösse }\end{array}$ & $\begin{array}{l}\text { Integration von } \\
\text { ERP-Modulen }\end{array}$ & Finanzwirtschaft & 0.007 & $1.0 \%$ \\
\hline $1 b$ & & & Warenwirtschaft & 0.006 & $1.0 \%$ \\
\hline $1 b$ & & & Einkauf & 0.024 & $5.0 \%$ \\
\hline $1 b$ & & & Controlling und FIS & 0.044 & $5.0 \%$ \\
\hline $2 \mathrm{a}$ & $\begin{array}{l}\text { Umsatzent- } \\
\text { wicklung }\end{array}$ & $\begin{array}{l}\text { Einsatz von } \\
\text { ERP-Modulen }\end{array}$ & $\begin{array}{l}\text { Vertrieb und } \\
\text { Auftragsabwicklung }\end{array}$ & 0.041 & $5.0 \%$ \\
\hline$*)$ & \multicolumn{5}{|c|}{ Asymptotische Signifikanz (zweiseitig) im Chi-Quadrat-Test nach Pearson } \\
\hline$* *)$ & \multicolumn{5}{|c|}{ Verbleibende Irrtumswahrscheinlichkeit $\alpha$ im zweiseitigen Test } \\
\hline
\end{tabular}

Tabelle 3: Signifikante Teilergebnisse der Zusammenhangsanalyse zwischen den Merkmalen Unternehmensgrösse und Umsatzentwicklung und dem Einsatz und der Integration von ERP- und E-Business-Modulen

\section{Hypothese 2}

Hypothese 2 untersucht den Zusammenhang zwischen der Umsatzentwicklung und dem Einsatz und der Integration von ERP- und E-Business-Modulen. Die Umsatzentwicklung der Unternehmen wird daran gemessen, wie sich der Umsatz in den letzten drei Jahren $(2001$ - 2003) im Grossen und Ganzen entwickelte. Die Antworten werden in fünf Klassen eingeteilt: (1) stark gesunken, (2) gesunken, (3) stabil (stagnierend), (4) gestiegen und (5) stark gestiegen. 
Die Umsatzentwicklung als Determinante von ERP-Einsatz (Hypothese 2a) und ERP-Integration (Hypothese 2b) zeigt einen signifikanten Einfluss nur auf den Einsatz des Moduls Vertrieb und Auftragsabwicklung (Tabelle 3). Je besser die Umsatzentwicklung eines Unternehmens ist, desto häufiger werden ERP-Module zur Unterstützung von Vertrieb und Auftragsabwicklung eingesetzt. Bei vielen anderen Modulen ist der Test wegen zu geringer Erwartungshäufigkeiten nicht anwendbar. ${ }^{4}$ Dies gilt auch für den Zusammenhang zwischen der Umsatzentwicklung und der Integration von ERP-Modulen.

Der Zusammenhang zwischen der Umsatzentwicklung und dem Einsatz und der Integration von E-Business-Modulen kann nicht untersucht werden. In allen Fällen sind die Erwartungshäufigkeiten zu gering, um den Test anzuwenden.

\section{Hypothese 3}

In Hypothese 3 bilden der Einsatz (Hypothese 3a) und die Integration (Hypothese 3b) von ERP- und E-Business-Modulen den exogenen Faktor. Geprüft wird, ob aus dem Einsatz oder der Integration dieser Module positive Auswirkungen auf den Unternehmenserfolg resultieren. Für die Messung des Unternehmenserfolgs wird die Umsatzentwicklung eines Unternehmens mit der Umsatzentwicklung der betreffenden Branche in Verbindung gebracht. Dabei gehen die Umsatzentwicklung des Unternehmens und die Umsatzentwicklung im Vergleich zur Branche zu gleichen Teilen in die Bewertung ein. Der daraus entstehende Bewertungsindex für den Unternehmenserfolg wird für die Assoziationsanalyse wie folgt klassiert: (1) weniger erfolgreich, (2) erfolgreich, (3) sehr erfolgreich.

Ein signifikanter Einfluss auf den Unternehmenserfolg kann nur im Falle der Integration des ERP-Moduls „Betriebsdatenerfassung“ beobachtet werden (Tabelle 4). Unternehmen, deren Betriebsdatenerfassung in ein ERP-System integriert ist, sind hoch signifikant häufiger erfolgreich als andere Unternehmen.

$4 \quad$ Dies trifft zu auf die Module Finanzwirtschaft, Einkauf, Service und Dienstleistungen, Produktionsplanung- und-steuerung, Betriebsdatenerfassung, Personalwirtschaft und Controlling/FIS. 


\begin{tabular}{llllll}
\hline $\begin{array}{l}\text { Hypo } \\
\text { these }\end{array}$ & Merkmal 1 & Modul & Merkmal 2 & $\begin{array}{l}\text { Signifi- } \\
\text { kanz *) }\end{array}$ & $\begin{array}{l}\text { Signifi- } \\
\text { kanz- } \\
\text { niveau **) }\end{array}$ \\
\hline $3 b$ & $\begin{array}{l}\text { Integration von } \\
\text { ERP-Modulen }\end{array}$ & $\begin{array}{l}\text { Betriebsdaten- } \\
\text { erfassung }\end{array}$ & $\begin{array}{l}\text { Erfolg des } \\
\text { Unternehmens }\end{array}$ & 0.004 & $1.0 \%$ \\
\hline$*)$ & Asymptotische Signifikanz (zweiseitig) im Chi-Quadrat-Test nach Pearson \\
$* *)$ & Verbleibende Irrtumswahrscheinlichkeit $\alpha$ im zweiseitigen Test \\
\hline
\end{tabular}

Tabelle 4: Signifikante Teilergebnisse der Zusammenhangsanalyse zwischen den Merkmalen Einsatz und Integration von ERP-und E-Business-Modulen und dem Erfolg des Unternehmens

Der Zusammenhang zwischen dem Unternehmenserfolg und den E-BusinessModulen kann nur im Falle des Einsatzes der Module E-Commerce und Branchensoftware sowie im Fall der Integration des Moduls Branchensoftware analysiert werden. Diese Zusammenhänge sind nicht signifikant. Der Einsatz von ECommerce- und Branchensoftware wirkt sich folglich nicht signifikant auf den Unternehmenserfolg aus. Dies gilt auch für die Integration von Branchensoftware. Die Hypothese, der Einsatz und die Integration von E-Business-Modulen wirke sich positiv auf den Unternehmenserfolg aus, kann damit nicht bestätigt werden.

\section{Zusammenfassung und Ausblick}

Insgesamt machen die Untersuchungsergebnisse deutlich, dass Schweizer KMU sehr hohe Ansprüche an Business Software stellen, allen voran die Anpassungsfähigkeit der Systeme und die Kundennähe des Anbieters. Die Schwerpunkte der Integration von Business Software werden weiterhin im unternehmensinternen Bereich liegen. Als ein anhaltender Trend erweist sich der Auf- und Ausbau von EBusiness-Applikationen, in dessen Rahmen die KMU das Thema der unternehmensübergreifenden Integration verstärkt angehen werden. Die regionalen Schweizer Anbieter haben deshalb einen Wettbewerbsvorteil, wenn sie ERPSysteme anbieten, die neben der Integration der Basismodule auch die Integration von E-Business-Modulen vorsehen.

Für die KMU bringt das zunehmende Angebot solcher Lösungen die grosse Chance, integrierte Lösungen mit einem überschaubaren Aufwand umsetzen zu können. Tendenziell wird dies in zunehmendem Masse auch für die kleinen unter den KMU gelten. Heute hängt der Umfang der Integration noch sehr stark von der Unternehmensgrösse ab. Die Module Finanzwirtschaft, Warenwirtschaft, Einkauf und Controlling sind in den grösseren KMU signifikant häufiger in ein Gesamt- 
system integriert als in den kleineren KMU. Dies könnte sich in den nächsten Jahren ändern.

\section{Literatur}

[ $\mathrm{Bac}^{+}$96] Backhaus, K. et al.: Multivariate Analysemethoden. Eine anwendungsorientierte Einführung. 8. Aufl., Springer: Berlin et al., 1996.

[Buh $\left.{ }^{+} 01\right]$ Buhl, L.; Christ, J.; Pape, U.: Marktstudie. Softwaresysteme für Enterprise Application Integration. In: Dangelmaier, W.; Bohner, M. (Hrsg.): ALB-HNI-Verlagsschriftenreihe, Band 7, Fraunhofer Anwendungszentrum für Logistikorientierte Betriebswirtschaft: Paderborn, 2001.

[Davy01] Davydov, M. M.: Corporate Portals and E-Business Integration. A Manager's Guide. McGraw-Hill, 2001.

[Dett02] Dettling, W.: EAI oder die Sehnsucht nach einer heilen Welt. In: Netzguide "Enterprise Application Integration", Oktober 2002, S. 7.

[Det ${ }^{+}$04] Dettling, W.; Leimstoll, U.; Schubert, P., Netzreport'5: Einsatz von Business Software in kleinen und mittleren Schweizer Unternehmen. Basel: Fachhochschule beider Basel (FHBB), Institut für angewandte Betriebsökonomie (IAB), Arbeitsbericht EBusiness Nr. 15, 2004.

[Dürs98] Dürselen, A.: Integrationspotentiale in kleinen und mittleren Unternehmen. Eul: Lohmar, Köln, 1998.

[Holt03] Holten, R.: Integration von Informationssystemen. In: Wirtschaftsinformatik 45, 2003, 1, S. 41-52.

[Kaib02] Kaib, M.: Enterprise Application Integration. Grundlagen, Integrationsprodukte, Anwendungsbeispiele. Deutscher Universitätsverlag: Wiesbaden, 2002.

[Kel102] Keller, W.: Enterprise Application Integration. Erfahrungen aus der Praxis. dpunkt, 2002.

[Leim01] Leimstoll, U.: Informationsmanagement in mittelständischen Unternehmen. Eine mikroökonomische und empirische Untersuchung. Frankfurt am Main et al.: Peter Lang, 2001.

[LePo98] Levy, M.; Powell, P.: SME flexibility and the role of information systems. In: Small Business Economics 11, 1998, S. 183-196.

[Lint01] Linthicum, D.: B2B Application Integration. Addison-Wesley Longman: Amsterdam, 2001.

[Lyba96] Lybaert, N.: Information. Erfolgsfaktor in industriellen KMU am Beispiel Belgiens. In: Internationales Gewerbearchiv 44, 1996, 1, S. 43-54. 
[Ray ${ }^{+}$95] Raymond, L.; Paré, G.; Bergeron F.: Matching information technology and organizational structure. An empirical study with implications for performance. In: European Journal of Information Systems 4, 1995, 1, S. 3-16.

[Sach92] Sachs, L.: Angewandte Statistik. Anwendung statistischer Methoden. 7. Aufl., Springer: Berlin et al., 1992.

[Sche91] Scheer, A.-W. (Hrsg.): CIM im Mittelstand. Fachtagung. Saarbrücken, 20.-21. Februar 1991. Springer: Berlin et al., 1991.

[Sche96] Scheer, A.-W.: Architekturen für das Information Engineering. In: Heilmann, H.; Heinrich, L. J.; Roithmayr, F. (Hrsg.): Information Engineering. Wirtschaftsinformatik im Schnittpunkt von Wirtschafts-, Sozial- und Ingenieurwissenschaften. Oldenbourg: München, Wien, 1996, S. 235-260.

[ScWi02] Schelp, J.; Winter, R.: Enterprise Portals und Enterprise Application Integration. Begriffsbestimmung und Integrationskonzeptionen. In: HMD, Praxis der Wirtschaftsinformatik, Nr. 225, Juni 2002, S. 6-19.

[Sch $\left.{ }^{+} 93\right]$ Scholl, M.; Lippold, H.; Hilgenfeldt J.: Vernetzung von IuK-Techniken. Chancen und Risiken der Informations- und Kommunikationstechniken für kleinere und mittlere Unternehmen. Vieweg: Braunschweig, Wiesbaden, 1993.

[ScDo02] Schopp, B.; Dold, D.: Beim Zauberwort EAI darf die Benutzersicht nicht vergessen werden. In: Netzguide "Enterprise Application Integration", Oktober 2002, S. 2224.

[Schu04] Schubert, P.: E-Business mit betriebswirtschaftlicher Standardsoftware. In: Schubert, P.; Wölfle, R.; Dettling, W. (Hrsg.): E-Business mit betriebswirtschaftlicher Standardsoftware. Einsatz von Business Software in der Praxis. Hanser: München, Wien 2004, S. $1-8$

[Sch $\left.{ }^{+} 03\right]$ Schubert, P.; Wölfle, R.; Dettling, W. (Hrsg.): E-Business-Integration. Fallstudien zur Optimierung elektronischer Geschäftsprozesse. Hanser: München, Wien, 2003.

[Stei93] Steinborn, D.: Die Analyse nominal-skalierter Daten in Kontingenztafeln mit Assoziationsmaßen unter besonderer Berücksichtigung von Datenvariationen. Lang: Frankfurt am Main et al., 1993.

[VoZe02] Voigtmann, P.; Zeller, T. (2002): Enterprise Application Integration und B2B Integration im Kontext von Electronic Business und Elektronischen Marktplätzen. Teil 1: Grundlagen und Anforderungen. FORWIN-Bericht, FWN-2002-013, Nürnberg, 2002. 